\title{
Robust Global Stabilization of Spacecraft Rendezvous System via Gain Scheduling
}

\author{
Qian Wang Guang-Ren Duan \\ Center for Control Theory and Guidance Technology, Harbin Institute of Technology, Harbin 150001, China
}

\begin{abstract}
The problem of robust global stabilization of a spacecraft circular orbit rendezvous system with input saturation and inputadditive uncertainties is studied in this paper. The relative models with saturation nonlinearity are established based on ClohesseyWiltshire equation. Considering the advantages of the recently developed parametric Lyapunov equation-based low gain feedback design method and an existing high gain scheduling technique, a new robust gain scheduling controller is proposed to solve the robust global stabilization problem. To apply the proposed gain scheduling approaches, only a scalar nonlinear equation is required to be solved. Different from the controller design, simulations have been carried out directly on the nonlinear model of the spacecraft rendezvous operation instead of a linearized one. The effectiveness of the proposed approach is shown.
\end{abstract}

Keywords: Robust global stabilization, spacecraft rendezvous system, parametric Lyapunov equation, input saturation, inputadditive uncertainties, gain scheduling.

\section{Introduction}

Spacecraft rendezvous is an important technology for present and future space missions. Successful rendezvous is the precondition of many astronautic missions, such as repairing, intercepting, docking, saving, large-scale structure assembling and satellite networking ${ }^{[1]}$. Considering a target spacecraft in a circular orbit and another chaser spacecraft in its neighborhood, the relative motion between the two neighboring spacecraft can be described by autonomous nonlinear differential equations. If the distance between them is much smaller than the orbit radius, the relative dynamics is closely approximated by $\mathrm{C}-\mathrm{W}$ equation, derived by Clohessey and Wiltshire in $1960^{[2]}$. During the last few decades, the spacecraft rendezvous has been actively studied and many results in control theory and technologies have been developed. Many advanced methods have been used to solve the rendezvous control problem. For example, the model predictive control approach was developed for spacecraft rendezvous ${ }^{[3-7]}$; adaption control theory was applied to the rendezvous and docking problem ${ }^{[8]}$; in [9] the problem of rendezvous was cast into a stabilization problem analyzed by Lyapunov theory; the neural-network-based controller was proposed for rendezvous maneuvers ${ }^{[10]}$; in [11], an adaptive backstepping control law was designed for spacecraft in proximity operation missions and a parametric Lyapunov differential equation approach to solve rendezvous with control constraints ${ }^{[12]}$.

For astronautic missions, the control input constraint is an important issue we must pay attention to. In practical engineering, the orbital control input force is limited due to

\footnotetext{
Regular paper

Manuscript received March 25, 2013; revised November 21, 2013

This work was supported by the Innovative Team Program of the National Natural Science Foundation of China (No.61021002) and National Basic Research Program of China (973 Program) (No. 2012CB821205).
}

the constraints of the thrust equipment, the limited quality of fuel and the power the engine provided, etc. Among the constraints, the constraint from the thrust provided by the thrust equipment is very important. So far, there are some results for this problem. For instance, [13] and [14] studied the lowest energy controller with fixed time. Because of using the optimal control theory, in order to realize the proposed controller, the association state differential equation need to be solved online. The spacecraft rendezvous system with chance-constrained and disturbance was studied by model predictive control in [7]. For more information in both theory and practice, see the references cited therein.

In addition, the possible mass variation or the errors of the thrusters may bring the inaccuracy to the control input, which can be regarded as the uncertainty of the input. These uncertainties may degrade the precision, the stability or even the safety of the rendezvous missions. Recent years, there have been many studies on the systems with inputadditive uncertainties ${ }^{[15,16]}$. However, the input-additive uncertainties have not attracted enough attentions in the existing studies on spacecraft rendezvous control problems. This leads to our desire to take these uncertainties into consideration.

The gain-scheduling approach is perhaps one of the most popular non-linear control design approaches which has been widely applied in the fields of aerospace. It employs powerful linear design tools on difficult nonlinear problems. Gain scheduling does not require severe structural assumptions on the plant model and the approach can be used in the absence of complete, analytical plant models. Design by gain scheduling may preserve well-understood linear intuition and is carried out using the physical variables in the plant model ${ }^{[17,18]}$. In recent years, some results have been reported to deal with the control problem of spacecraft rendezvous; see [19] and the references therein. There are basically three kinds of scheduling approaches, namely 
the continuous static scheduling approach, the continuous dynamic scheduling approach, and the discrete scheduling approach. In the continuous static scheduling approach, the feedback gains are scheduled continuously according to solutions to some algebraic equations or inequalities in terms of the state vectors. The continuous eigenvalue assignment approach $^{[20]}$, the gain scheduling approach in [21] (see also [15]), and the gain scheduling approach proposed in [22] fall into this category. The main difficulty existing in this class of approaches is that some complex nonlinear equations should be solved online for each time instant $t$. In the continuous dynamic scheduling approach, the feedback gains are scheduled continuously according to solutions to some differential equations in terms of the state vectors. Examples of this class of scheduling approaches can be found in $[23-25]$ and the references therein. Different from the continuous scheduling scheme, the discrete scheduling approach changes the feedback gains in some discrete times. This approach is also known as piecewise-linear feedback. Examples can be found in [26-28]. Such approaches are easy to implement. However, the resulting controller is discontinuous and may cause some practical problem ${ }^{[22]}$.

In this paper, we develop an effective nonlinear continuous static gain scheduling controller to solve the global stabilization problem of the circular orbit rendezvous system subject to input saturation and input-additive uncertainties. With independent continuous control accelerations used as the control signals to $\mathrm{C}-\mathrm{W}$ equations, we establish the relative motion equation with input saturation and input-additive uncertainties. By using the parametric Lyapunov equation-based low gain feedback design method and an existing high gain scheduling technique, a new robust gain scheduling controller is proposed for the orbit rendezvous system.

The rest of this paper is organized as follows. Section 2 presents the dynamic model of spacecraft rendezvous, and formulates the robust global stabilization problem. In Section 3, the robust gain scheduling controller is proposed to solve the global control problem for the spacecraft rendezvous system. Then, the numerical simulation is given to illustrate the applicability of the presented approach in Section 4. Finally, Section 5 concludes the paper.

Notations. Throughout the paper, the notation used is fairly standard. We use $A^{\mathrm{T}}, \operatorname{tr}(A), \lambda(A), \lambda_{\max }(A)$ and $\lambda_{\min }(A)$ to denote the transpose, the trace, the set of eigenvalues, the maximal eigenvalue and the minimal eigenvalue of matrix $A$, respectively. $\operatorname{diag}\{\cdot\}$ stands for a block-diagonal matrix. $I$ denotes the identity matrix with compatible dimensions. For a real symmetric matrix $P$, the notation $P>0(P<0)$ is used to denote its positive (negative) definiteness. The function $\operatorname{sgn}$ is defined $\operatorname{as} \operatorname{sgn}(y)=1$ if $y \geqslant 0$ and $\operatorname{sgn}(y)=-1$ if $y<0$. The saturation function in this paper is

$$
\operatorname{sat}_{\alpha}(u)= \begin{cases}-\alpha, & u<-\alpha \\ u, & |u| \leqslant \alpha \\ \alpha, & u>\alpha\end{cases}
$$

where $\alpha>0$ is the saturation level and standard saturation function $\operatorname{sat}_{1}(u)$ will be denoted by $\operatorname{sat}(u)$ for short. When $u$ is a vector, with slight abuse of notation, the absolute value is applied element-wise. Finally, for a vector $\boldsymbol{x} \in \mathbf{R}^{m}$, we denote $\|\boldsymbol{x}\|_{\infty}=\max _{1 \leqslant i \leqslant m}\left|\boldsymbol{x}_{i}\right|$, where $\boldsymbol{x}_{i}$ is the $i$-th element of $\boldsymbol{x}$.

\section{Dynamic model and problem formu- lation}

In this section, the relative motion dynamics is analyzed based on the $\mathrm{C}-\mathrm{W}$ equation ${ }^{[2]}$. By using input accelerations in the three directions as control input, we establish the relative model for orbit rendezvous system with input saturation and input-additive uncertainties. Finally, the control problems which are concerned in this paper are presented.

\subsection{Description of relative motion}

The spacecraft rendezvous system is illustrated in Fig. 1. We assume that the two spacecraft (the target and chaser) are adjacent, and that the target spacecraft is in a circular orbit whose radius is $R$. Assume that the vector from the target spacecraft to the chaser spacecraft is denoted by $r$. The right-handed coordinate system (rotating coordinate system) $O-X Y Z$ is fixed at the center of mass of the target with $X$ axis along the radial direction, $Y$ axis along the flight direction of the target, and $Z$ axis out of the orbit plane, respectively. Denote the gravitational parameter $\mu=G M$, where $M$ is the mass of the center planet and $G$ is the gravitational constant. Then the orbit rate of the target orbit is given by $n=\mu^{\frac{1}{2}} / R^{\frac{3}{2}}$.

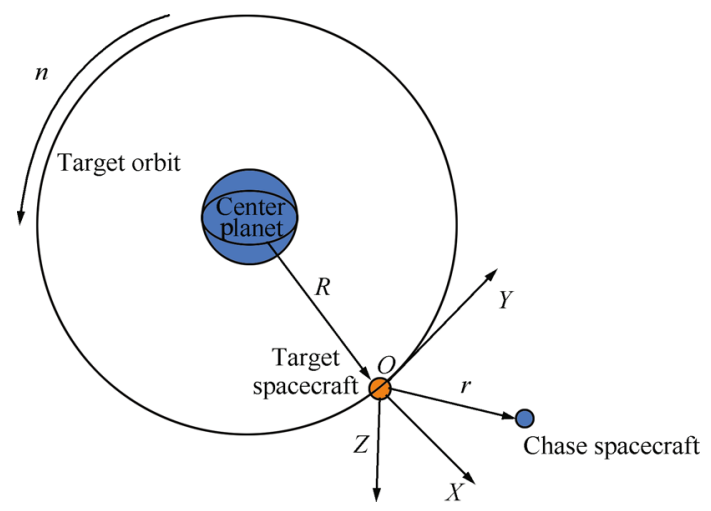

Fig. 1 Circular orbit and coordinate system

Considering the input constraint and input-additive uncertainties, the relative motion between the target and chaser can be governed by Newton's equations ${ }^{[29]}$

$$
\left\{\begin{array}{l}
\ddot{x}=2 n \dot{y}+n^{2}(R+x)-\rho \mu(R+x)+\operatorname{sat}_{\alpha_{X}}\left(a_{x}+g_{1}\right) \\
\ddot{y}=-2 n \dot{x}+n^{2} y-\rho \mu y+\operatorname{sat}_{\alpha_{Y}}\left(a_{y}+g_{2}\right) \\
\ddot{z}=-\rho \mu z+\operatorname{sat}_{\alpha_{Z}}\left(a_{z}+g_{3}\right)
\end{array}\right.
$$

where $\rho=\left((R+x)^{2}+y^{2}+z^{2}\right)^{-\frac{3}{2}} ; a_{x}, a_{y}$ and $a_{z}$ are respectively the control input accelerations in the three directions; 
$\alpha_{X}, \alpha_{Y}$ and $\alpha_{Z}$ are respectively the maximal accelerations that the thrusts can generate in the three directions; $g_{1}, g_{2}$ and $g_{3}$ are respectively the input uncertainties in the three directions.

By linearizing (2) around the center of mass of the target, we obtain

$$
\left\{\begin{array}{l}
\ddot{x}=2 n \dot{y}+3 n^{2} x+\operatorname{sat}_{\alpha_{X}}\left(a_{x}+g_{1}\right) \\
\ddot{y}=-2 n \dot{x}+\operatorname{sat}_{\alpha_{Y}}\left(a_{y}+g_{2}\right) \\
\ddot{z}=-n^{2} z+\operatorname{sat}_{\alpha_{Z}}\left(a_{z}+g_{3}\right)
\end{array}\right.
$$

which is known as the Hill's equation or Clohessy-Wiltshire equation $^{[2]}$.

Notice that we denote $D=\operatorname{diag}\left\{\alpha_{X}, \alpha_{Y}, \alpha_{Z}\right\}$,

$$
\boldsymbol{a}=\left[\begin{array}{lll}
a_{x} & a_{y} & a_{z}
\end{array}\right]^{\mathrm{T}}
$$

and

$$
\boldsymbol{g}^{*}=\left[\begin{array}{lll}
g_{1} & g_{2} & g_{3}
\end{array}\right]^{\mathrm{T}}
$$

where $\boldsymbol{a}$ is the acceleration vector due to thrust forces on the chaser, and $\boldsymbol{g}^{*}$ is the input uncertainties vector.

We set

$$
\begin{aligned}
& \boldsymbol{u}^{\prime}(t) \triangleq\left[\begin{array}{lll}
\operatorname{sat}_{\alpha_{X}}\left(a_{x}\right) & \operatorname{sat}_{\alpha_{Y}}\left(a_{y}\right) & \operatorname{sat}_{\alpha_{Z}}\left(a_{z}\right)
\end{array}\right]^{\mathrm{T}}= \\
& D\left[\begin{array}{lll}
\operatorname{sat}\left(\frac{a_{x}}{\alpha_{X}}\right) & \operatorname{sat}\left(\frac{a_{y}}{\alpha_{Y}}\right) & \operatorname{sat}\left(\frac{a_{z}}{\alpha_{Z}}\right)
\end{array}\right]^{\mathrm{T}}= \\
& D \operatorname{Dsat}\left(D^{-1} \boldsymbol{a}\right) .
\end{aligned}
$$

By choosing the state vector

$$
\boldsymbol{x}=\left[\begin{array}{llllll}
x & y & z & \dot{x} & \dot{y} & \dot{z}
\end{array}\right]^{\mathrm{T}}
$$

the relative motion equation with input uncertainties can be described as

$$
\dot{\boldsymbol{x}}=A \boldsymbol{x}+B \operatorname{sat}(\boldsymbol{u}+\boldsymbol{g}(\boldsymbol{x}, t))
$$

where $\boldsymbol{u}=D^{-1} \boldsymbol{a}, \boldsymbol{g}(\boldsymbol{x}, t)=D^{-1} \boldsymbol{g}^{*}(\boldsymbol{x}, t)$

$$
\begin{aligned}
A & =\left[\begin{array}{cccccc}
0 & 0 & 0 & 1 & 0 & 0 \\
0 & 0 & 0 & 0 & 1 & 0 \\
0 & 0 & 0 & 0 & 0 & 1 \\
3 n^{2} & 0 & 0 & 0 & 2 n & 0 \\
0 & 0 & 0 & -2 n & 0 & 0 \\
0 & 0 & -n^{2} & 0 & 0 & 0
\end{array}\right] \\
B & =\left[\begin{array}{lll}
0 & 0 & 0 \\
0 & 0 & 0 \\
0 & 0 & 0 \\
1 & 0 & 0 \\
0 & 1 & 0 \\
0 & 0 & 1
\end{array}\right] D
\end{aligned}
$$

and $\boldsymbol{g}^{*}(\boldsymbol{x}, t)$ is the input uncertainties vector, sat : $\mathbf{R}^{3} \rightarrow$ $\mathbf{R}^{3}$ is a standard saturation function. The saturation function is defined in [16] and recalled as follows.

Definition 1. Function $\sigma: \mathbf{R}^{m} \rightarrow \mathbf{R}^{m}$ is called a saturation function, if

1) $\sigma(u)$ is decentralized, i.e., $\sigma(u)=\left[\sigma_{1}\left(u_{1}\right), \cdots, \sigma_{m}\left(u_{m}\right)\right]^{\mathrm{T}}$ for each $i=1$ to $m$;

2) $\sigma_{i}$ is locally Lipschitz;

3) $s \sigma_{i}(s)>0$ whenever $s \neq 0$;

4) $\min \left\{\lim _{s \rightarrow 0^{+}}\left(\sigma_{i}(s) / s\right), \lim _{s \rightarrow 0^{-}}\left(\sigma_{i}(s) / s\right)\right\}>0$;

5) $\liminf |s| \rightarrow \infty\left|\sigma_{i}(s)\right|>0$.

Remark 1. It follows from Definition 1 that the standard saturation function $\operatorname{sat}(s)=\operatorname{sgn}(s) \min \{|s|, 1\}$ is a saturation function. So, it has the properties of the saturation function.

Proposition 1. The matrix pair $(A, B)$ is controllable and all the eigenvalues of $A$ are on the imaginary axis, namely, $(A, B)$ is asymptotically null controllable with bounded controls (ANCBC).

Roughly speaking, ANCBC means that any fixed bounded initial state can be steered to the origin with bounded controls.

Regarding the uncertain vector, we only require knowing an upper bound on the norm of $\boldsymbol{g}^{*}$.

Assumption 1. ${ }^{[15]}$ The uncertain vector is piecewise continuous in $t$, locally Lipschitz in $\boldsymbol{x}$ and its norm is bounded by a known function:

$$
\left\|\boldsymbol{g}^{*}(\boldsymbol{x}, t)\right\| \leqslant g_{0}\left(\|\boldsymbol{x}\|_{\infty}\right), \quad \forall(\boldsymbol{x}, t) \in \mathbf{R}^{n} \times \mathbf{R}_{+}
$$

where function $g_{0}: \mathbf{R}_{+} \rightarrow \mathbf{R}_{+}$is known and is locally Lipschitz with $g_{0}(0)=0$. Without loss of generality, we also assume that $g_{0}$ is nondecreasing.

\subsection{Problem formulation}

The whole rendezvous process can be described by the transformation of state vectors $\boldsymbol{x}(t)$ from nonzero initial states $\boldsymbol{x}\left(t_{0}\right)$ to the terminal states $\boldsymbol{x}\left(t_{f}\right)=0$, where $t_{f}$ is the rendezvous time ${ }^{[12]}$. In this paper, we will propose a nonlinear continuous static gain scheduled feedback law to achieve the rendezvous mission in the presence of actuator saturation and input uncertainty.

Problem 1. Design a nonlinear gain scheduling controller $\boldsymbol{u}(\boldsymbol{x})$ for system (4), with Proposition $1, g(\boldsymbol{x}, t)$ satisfying Assumption 1, such that the closed-loop system is globally asymptotically stable.

\section{Controller design}

In this section, we construct a continuous static gain scheduled feedback law that solves Problem 1 as formulated in the previous section. The construct of the gain scheduled feedback law is based on the low-and-high gain $\operatorname{design}^{[30]}$. There are three methods to design low gain feedback: eigenstructure assignment based algorithm ${ }^{[31]}$, algebraic Riccati equation (ARE) based approach ${ }^{[32,33]}$ and a parametric Lyapunov equation based method ${ }^{[34]}$. The parametric Lyapunov equation based approach possesses the advantages of both the eigenstructure assignment approach and the ARE-based approach ${ }^{[34]}$. Firstly, the parametric Lyapunov equation is conceptually appealing and directly results in a quadratic Lyapunov function for the closed-loop system. Secondly, it avoids the numerical stiffness encountered in the solution of an ARE with a small value of the low gain parameter and the structural decomposition of the 
open loop system required for the eigenstructure assignment. In this paper, we choose the parametric Lyapunovbased method to design the gain scheduled feedback law. For the parametric Lyapunov-based approach, matrix $P(\gamma)$ is the unique positive definite solution to the following parametric ARE

$$
P A+A^{\mathrm{T}} P-P B B^{\mathrm{T}} P=-\gamma P
$$

where $\gamma>0$ is the design parameter, and all the eigenvalues of $A$ are assumed to be on the imaginary axis.

Properties of solutions to the ARE (7) are summarized in Lemma 1 below.

Lemma $\mathbf{1}^{[34]}$. Assume that $(A, B)$ is controllable and $A$ has all its eigenvalues on the imaginary axis. Then,

1) there exists a unique matrix $P(\gamma)>0$ which solves the $\operatorname{ARE}(7), P(\gamma)=W^{-1}(\gamma)$, where $W(\gamma)$ is the unique positive-definite solution to the following Lyapunov matrix equation

$$
W\left(A+\frac{\gamma}{2} I\right)^{\mathrm{T}}+\left(A+\frac{\gamma}{2} I\right) W=B B^{\mathrm{T}}
$$

2) matrix $A-B B^{\mathrm{T}} P(\gamma)$ is Hurwitz;

3) $\lim _{r \rightarrow 0} P(\gamma)=0$;

4) $P(\gamma)$ is continuously differentiable and strictly increasing with respect to $\gamma$, i.e., $\mathrm{d} P(\gamma) / \mathrm{d} \gamma>0$;

5) $\operatorname{tr}\left(B^{\mathrm{T}} P(\gamma) B\right)=n \gamma$, where $n$ is the dimension of the system matrix $A$.

Before giving the main results, we define two invariant ellipsoid sets:

$$
\begin{aligned}
& \mathscr{E}=\left\{\boldsymbol{x} \in \mathbf{R}^{6}: 6 \gamma(\boldsymbol{x}) \boldsymbol{x}^{\mathrm{T}} P(\gamma(\boldsymbol{x})) \boldsymbol{x} \leqslant 1, \gamma(\boldsymbol{x})=\gamma^{*}\right\} \\
& \mathscr{L}=\left\{\boldsymbol{x} \in \mathbf{R}^{6}: 6 \gamma(\boldsymbol{x}) \boldsymbol{x}^{\mathrm{T}} P(\gamma(\boldsymbol{x})) \boldsymbol{x} \leqslant 1, \gamma(\boldsymbol{x}) \in\left(0, \gamma^{*}\right)\right\} .
\end{aligned}
$$

Theorem 1. Let Assumption 1 hold, $P(\gamma(\boldsymbol{x}))$ be the unique positive definite solution to the ARE (7). Then the gain scheduled state feedback controller

$$
\boldsymbol{u}(\boldsymbol{x})= \begin{cases}-(1+\eta(\boldsymbol{x})) B^{\mathrm{T}} P(\gamma(\boldsymbol{x})) \boldsymbol{x}, & \text { if } \boldsymbol{x} \in \mathscr{L} \\ -(1+\eta(\boldsymbol{x})) B^{\mathrm{T}} P\left(\gamma^{*}\right) \boldsymbol{x}, & \text { if } \boldsymbol{x} \in \mathscr{E}\end{cases}
$$

solves Problem 1, where

$$
\begin{gathered}
\gamma(\boldsymbol{x})=\max \left\{\gamma \in\left(0, \gamma^{*}\right]:\left(\boldsymbol{x}^{\mathrm{T}} P(\gamma) \boldsymbol{x}\right) \operatorname{tr}\left(B^{\mathrm{T}} P(\gamma) B\right) \leqslant 1\right\} \\
\eta(\boldsymbol{x})=\frac{\eta_{0}\left(g^{\prime}\left(\|\boldsymbol{x}\|_{\infty}\right)^{2}+0.1\right) \varphi\left(2 g_{0}\left(\|\boldsymbol{x}\|_{\infty}\right)+1\right)}{\gamma(\boldsymbol{x})}
\end{gathered}
$$

in which $\eta_{0}>12$ is a design parameter whose value is to be tuned according to the set of data admissible for state feedback; $g^{\prime}: \mathbf{R}_{+} \rightarrow \mathbf{R}_{+}$is any locally Lipschitz function that satisfies $g^{\prime}(s) \geqslant g_{0}(s) / s, \forall s>0 ; \varphi: \mathbf{R}_{+} \rightarrow \mathbf{R}_{+}$is locally Lipschiz and nondecreasing; the ellipsoid $\mathscr{E}$ and $\mathscr{L}$ are defined by (9) and (10), respectively.

Regarding function $\gamma(\boldsymbol{x})$, the following lemma was proved in [21].

Lemma 2. $\gamma(\boldsymbol{x})$ is a continuous function of $\boldsymbol{x} \in \mathbf{R}^{n}$ and is continuously differentiable in a neighborhood of any point $\boldsymbol{x}$ such that $0<\gamma(\boldsymbol{x})<\gamma^{*}$. Moreover, function $B^{\mathrm{T}} P(\gamma(\boldsymbol{x})) \boldsymbol{x}$ is globally Lipschitz.

Remark 2. The designed controller asymptotically stabilizes system (4) in the following way: the nonlinear controller steers the system trajectories to the neighbourhood of the origin $\mathscr{E}$, then the linear controller $-(1+$ $\eta(\boldsymbol{x})) B^{\mathrm{T}} P\left(\gamma^{*}\right) \boldsymbol{x}$ will asymptotically take the state to the origin. Such an idea was also used in the early literature to design global stabilizing controllers ${ }^{[20,21]}$. The proposed approach in this paper can obtain analytical expression of the controller and lead to good dynamic performance of the closed-loop system.

Proof. For simplicity, we denote $\boldsymbol{g}(\boldsymbol{x}, t)$ as $\boldsymbol{g}$.

When $\boldsymbol{x} \in \mathscr{L}$, we have

$$
\boldsymbol{u}(\boldsymbol{x})=-(1+\eta(\boldsymbol{x})) B^{\mathrm{T}} P(\gamma(\boldsymbol{x})) \boldsymbol{x} .
$$

We write the closed-loop system as

$$
\dot{\boldsymbol{x}}=A \boldsymbol{x}+B \operatorname{sat}\left(-(1+\eta(\boldsymbol{x})) B^{\mathrm{T}} P(\gamma(\boldsymbol{x})) \boldsymbol{x}+\boldsymbol{g}\right)
$$

whose right-hand side, in view of Lemma 2, is locally Lipschitz in $\boldsymbol{x}$ and piecewise continuous in $t$. For the closed-loop system, we choose the Lyapunov function

$$
V=\boldsymbol{x}^{\mathrm{T}} P(\gamma(\boldsymbol{x})) \boldsymbol{x} .
$$

By Definition 1, Remark 1, ARE (7) and

$$
\begin{gathered}
\left\|B^{\mathrm{T}} P(\gamma(\boldsymbol{x})) \boldsymbol{x}\right\|_{2}^{2} \leqslant\left\|B^{\mathrm{T}} P^{\frac{1}{2}}(\gamma(\boldsymbol{x}))\right\|_{2}^{2}\left\|P^{\frac{1}{2}}(\gamma(\boldsymbol{x})) \boldsymbol{x}\right\|_{2}^{2}= \\
\lambda_{\max }\left(B^{\mathrm{T}} P(\gamma(\boldsymbol{x})) B\right)\left(\boldsymbol{x}^{\mathrm{T}} P(\gamma(\boldsymbol{x})) \boldsymbol{x}\right) \leqslant \\
\operatorname{tr}\left(B^{\mathrm{T}} P(\gamma(\boldsymbol{x})) B\right)\left(\boldsymbol{x}^{\mathrm{T}} P(\gamma(\boldsymbol{x})) \boldsymbol{x}\right) \leqslant 1
\end{gathered}
$$

the evaluation of $V$ along the trajectories of system (14) is

$$
\begin{aligned}
\dot{V}= & 2 \boldsymbol{x}^{\mathrm{T}} P(\gamma(\boldsymbol{x})) \dot{\boldsymbol{x}}+\boldsymbol{x}^{\mathrm{T}} \frac{\mathrm{d} P(\gamma(\boldsymbol{x}))}{\mathrm{d} t} \boldsymbol{x}= \\
& \boldsymbol{x}^{\mathrm{T}} \frac{\mathrm{d} P(\gamma(\boldsymbol{x}))}{\mathrm{d} t} \boldsymbol{x}+2 \boldsymbol{x}^{\mathrm{T}} P(\gamma(\boldsymbol{x})) \\
& \left(A \boldsymbol{x}+B \operatorname{sat}\left(-(1+\eta(\boldsymbol{x})) B^{\mathrm{T}} P(\gamma(\boldsymbol{x})) \boldsymbol{x}+\boldsymbol{g}\right)\right)= \\
& \boldsymbol{x}^{\mathrm{T}}\left(P(\gamma(\boldsymbol{x})) A+A^{\mathrm{T}} P(\gamma(\boldsymbol{x}))\right) \boldsymbol{x}+\boldsymbol{x}^{\mathrm{T}} \frac{\mathrm{d} P(\gamma(\boldsymbol{x}))}{\mathrm{d} t} \boldsymbol{x}+ \\
& 2 \boldsymbol{x}^{\mathrm{T}} P(\gamma(\boldsymbol{x})) B \operatorname{sat}\left(-(1+\eta(\boldsymbol{x})) B^{\mathrm{T}} P(\gamma(\boldsymbol{x})) \boldsymbol{x}+\boldsymbol{g}\right)= \\
& \boldsymbol{x}^{\mathrm{T}} \frac{\mathrm{d} P(\gamma(\boldsymbol{x}))}{\mathrm{d} t} \boldsymbol{x}+2 \boldsymbol{x}^{\mathrm{T}} P(\gamma(\boldsymbol{x})) B \\
& \left(\operatorname{sat}\left(-(1+\eta(\boldsymbol{x})) B^{\mathrm{T}} P(\gamma(\boldsymbol{x})) \boldsymbol{x}+\boldsymbol{g}\right)+B^{\mathrm{T}} P(\gamma(\boldsymbol{x})) \boldsymbol{x}\right)- \\
& \boldsymbol{x}^{\mathrm{T}} \gamma(\boldsymbol{x}) P(\gamma(\boldsymbol{x})) \boldsymbol{x}-\boldsymbol{x}^{\mathrm{T}} P(\gamma(\boldsymbol{x})) B B^{\mathrm{T}} P(\gamma(\boldsymbol{x})) \boldsymbol{x} .
\end{aligned}
$$

Letting $\boldsymbol{\tau}=-B^{\mathrm{T}} P(\gamma(\boldsymbol{x})) \boldsymbol{x}$, where $\boldsymbol{\tau} \in \mathbf{R}^{3}$ and $v_{i}, g_{i}$ denote respectively the $i$-th element of $\boldsymbol{\tau}, \boldsymbol{g}$, we have

$$
\begin{aligned}
\dot{V} \leqslant & -\lambda_{\min }(\gamma(\boldsymbol{x}) P(\gamma(\boldsymbol{x}))) \boldsymbol{x}^{\mathrm{T}} \boldsymbol{x}- \\
& 2 \sum_{i=1}^{3} \tau_{i}\left(\operatorname{sat}\left((1+\eta(\boldsymbol{x})) \tau_{i}+g_{i}\right)-\operatorname{sat}\left(\tau_{i}\right)\right)+ \\
& \boldsymbol{x}^{\mathrm{T}} \frac{\mathrm{d} P(\gamma(\boldsymbol{x}))}{\mathrm{d} t} \boldsymbol{x} .
\end{aligned}
$$


According to the same procedure as used in $[15,16]$, it can be verified that

$$
-\tau_{i}\left(\operatorname{sat}\left((1+\eta(\boldsymbol{x})) \tau_{i}+g_{i}\right)-\operatorname{sat}\left(\tau_{i}\right)\right) \leqslant \frac{2\left|g_{i}\right|^{2} \varphi\left(2\left|g_{i}\right|\right)}{\eta(\boldsymbol{x})} .
$$

Hence, we conclude that

$$
\begin{aligned}
\dot{V} \leqslant & -\lambda_{\min }(\gamma(\boldsymbol{x}) P(\gamma(\boldsymbol{x}))) \boldsymbol{x}^{\mathrm{T}} \boldsymbol{x}+ \\
& \frac{12 g_{0}^{2}\left(\|\boldsymbol{x}\|_{\infty}\right) \varphi\left(2 g_{0}\left(\|\boldsymbol{x}\|_{\infty}\right)\right)}{\eta(x)}+\boldsymbol{x}^{\mathrm{T}} \frac{\mathrm{d} P(\gamma(\boldsymbol{x}))}{\mathrm{d} t} \boldsymbol{x} \leqslant \\
& -\lambda_{\min }(\gamma(\boldsymbol{x}) P(\gamma(\boldsymbol{x})))\left(1-\frac{4 m}{\eta_{0}}\right)\|\boldsymbol{x}\|_{\infty}^{2}+ \\
& \boldsymbol{x}^{\mathrm{T}} \frac{\mathrm{d} P(\gamma(\boldsymbol{x}))}{\mathrm{d} t} \boldsymbol{x} .
\end{aligned}
$$

By choosing

$$
\eta_{0}>4 m=12
$$

we have

$$
\dot{V}<\boldsymbol{x}^{\mathrm{T}} \frac{\mathrm{d} P(\gamma(\boldsymbol{x}))}{\mathrm{d} t} \boldsymbol{x} .
$$

According to the arguments used in [21], in view of (12), $\operatorname{tr}\left(B^{\mathrm{T}} P(\gamma) B\right)\left(\boldsymbol{x}^{\mathrm{T}} P(\gamma) \boldsymbol{x}\right)=1$, whenever $\gamma(\boldsymbol{x}) \neq \gamma^{*}$, i.e., whenever $P(\gamma(\boldsymbol{x}))$ is not a constant locally. Therefore, $\boldsymbol{x}^{\mathrm{T}} \frac{\mathrm{d} P(\gamma(\boldsymbol{x}))}{\mathrm{d} t} \boldsymbol{x}$ and $\dot{V}$ are either both zero or have opposite signs. So, for all $\eta_{0}$ satisfying (19), we have

$$
\dot{V}<0 \text {. }
$$

We can see the controller $\boldsymbol{u}(\boldsymbol{x})$ drives all the points $\boldsymbol{x} \in \mathscr{L}$ to $\mathscr{E}$.

When $\boldsymbol{x} \in \mathscr{E}$, we have

$$
\boldsymbol{u}(\boldsymbol{x})=-(1+\eta(\boldsymbol{x})) B^{\mathrm{T}} P\left(\gamma^{*}\right) \boldsymbol{x} .
$$

Considering system (4), we have the closed-loop system

$$
\dot{\boldsymbol{x}}=A \boldsymbol{x}-B \text { sat }\left((1+\eta(\boldsymbol{x})) B^{\mathrm{T}} P\left(\gamma^{*}\right) \boldsymbol{x}+\boldsymbol{g}\right), \forall \boldsymbol{x} \in \mathscr{E} .
$$

By choosing the Lyapunov function

$$
V(\boldsymbol{x})=\boldsymbol{x}^{\mathrm{T}} P\left(\gamma^{*}\right) \boldsymbol{x}
$$

the time derivative of $V(\boldsymbol{x})$ along the trajectories of system (23) can be evaluated as

$$
\begin{aligned}
& \dot{V}(\boldsymbol{x})=2 \boldsymbol{x}^{\mathrm{T}} P\left(\gamma^{*}\right) \dot{\boldsymbol{x}} \leqslant \\
& 2 \boldsymbol{x}^{\mathrm{T}} P\left(\gamma^{*}\right)\left(A \boldsymbol{x}+B \operatorname{sat}\left(-B^{\mathrm{T}} P\left(\gamma^{*}\right) \boldsymbol{x}+\boldsymbol{g}\right)\right) \leqslant
\end{aligned}
$$

$$
\begin{aligned}
& -\lambda_{\min }\left(\gamma^{*} P\left(\gamma^{*}\right)\right)\left(1-\frac{4 m}{\eta_{0}}\right)\|\boldsymbol{x}\|_{\infty}^{2}<0, \\
& \forall \boldsymbol{x} \neq 0, \eta_{0}>12 .
\end{aligned}
$$

So, all the trajectories arriving at $\mathscr{E}$ are driven asymptotically to the origin.

According to Proposition 1 and Lemma 1, $P(\gamma(\boldsymbol{x})) \rightarrow 0$ as $\gamma(\boldsymbol{x}) \rightarrow 0$. It follows that

$\mathscr{L}=\left\{\boldsymbol{x} \in \mathbf{R}^{6}: 6 \gamma(\boldsymbol{x}) \boldsymbol{x}^{\mathrm{T}} P(\gamma(\boldsymbol{x})) \boldsymbol{x} \leqslant 1, \gamma(\boldsymbol{x}) \in\left(0, \gamma^{*}\right)\right\}$

converges to the whole sets $\mathbf{R}^{6}$. So the controller (11) stabilizes system (4) globally.

\section{Simulation results}

In this section, we provide a numerical simulation to demonstrate the effectiveness and advantage of the proposed approach to the circular orbital rendezvous. Different from the controller design, our simulation will be carried out directly on the nonlinear plant described by (2). Thus, the angular velocity $n=7.2722 \times 10^{-5} \mathrm{rad} / \mathrm{s}$. For simulation purpose, we choose the initial condition in the target orbital coordinate system as

$$
x(0)=\left(\begin{array}{llllll}
1000 & 1000 & 800 & 5 & 3 & -1
\end{array}\right)^{\mathrm{T}}
$$

namely, the distances between the target and the chaser spacecraft in the three directions $X, Y$ and $Z$ are $1000 \mathrm{~m}$, $1000 \mathrm{~m}$, and $800 \mathrm{~m}$, respectively. The relative velocity are respectively $5 \mathrm{~m} / \mathrm{s}, 3 \mathrm{~m} / \mathrm{s}$ and $-1 \mathrm{~m} / \mathrm{s}$ in the three directions. Assume that the maximal accelerations supplied by the thrusts in the three directions satisfy $\left|\alpha_{X}\right| \leqslant 0.5$, $\left|\alpha_{Y}\right| \leqslant 0.5$ and $\left|\alpha_{Z}\right| \leqslant 0.1$, respectively. Let uncertainties $\boldsymbol{g}(\boldsymbol{x}, t)$ be such that $|\boldsymbol{g}(\boldsymbol{x}, t)| \leqslant 0.01\|\boldsymbol{x}\|_{\infty}+0.01\|\boldsymbol{x}\|_{\infty}^{2}$. So Assumption 1 is satisfied. Following Proposition 1 and Theorem 1, we can use the controller in (11) to stabilize system (2). With the above parameters we can compute the unique positive definite solution to the parametric ARE (7) as

$$
P=\left[\begin{array}{cccc}
P_{11} & 0 & P_{13} & 0 \\
0 & 100\left(2 n^{2}+\gamma^{2}\right) \gamma & 0 & 100 \gamma^{2} \\
P_{13}^{\mathrm{T}} & 0 & P_{33} & 0 \\
0 & 100 \gamma^{2} & 0 & 200 \gamma
\end{array}\right]
$$

in which $P_{11}, P_{13}$ and $P_{33}$ are respectively given in (28). By choosing $g^{\prime}(s)=0.01\|\boldsymbol{x}\|_{\infty}+0.01, \eta(x)$ is given by

$$
\eta(\boldsymbol{x})=\frac{2 \eta_{0}\left(\left(0.01\|\boldsymbol{x}\|_{\infty}+0.01\right)^{2}+0.1\right)}{\gamma(\boldsymbol{x})}
$$

here, we set $\eta_{0}=20$.

$$
P_{11}=\left[\begin{array}{cc}
\frac{4\left(2610 n^{10}+3073 \gamma^{2} n^{8}+1060 \gamma^{4} n^{6}+174 \gamma^{6} n^{4}+10 \gamma^{8} n^{2}+\gamma^{10}\right)}{\gamma\left(n^{2}+\gamma^{2}\right)\left(255 n^{6}+91 \gamma^{2} n^{4}+11 \gamma^{4} n^{2}+\gamma^{6}\right)} & \frac{-24 n^{3} \gamma^{2}\left(25 n^{4}+18 \gamma^{2} n^{2}+5 \gamma^{4}\right)}{255 n^{6}+91 \gamma^{2} n^{4}+11 \gamma^{4} n^{2}+\gamma^{6}} \\
\frac{-24 n^{3} \gamma^{2}\left(25 n^{4}+18 \gamma^{2} n^{2}+5 \gamma^{4}\right)}{255 n^{6}+91 \gamma^{2} n^{4}+11 \gamma^{4} n^{2}+\gamma^{6}} & \frac{4 \gamma^{3}\left(25 n^{4}+14 \gamma^{2} n^{2}+\gamma^{4}\right)\left(n^{2}+\gamma^{2}\right)}{255 n^{6}+91 \gamma^{2} n^{4}+11 \gamma^{4} n^{2}+\gamma^{6}}
\end{array}\right]
$$




$$
\begin{aligned}
& P_{13}=\left[\begin{array}{cc}
\frac{4 \gamma^{2}\left(\gamma^{2}+3 n^{2}\right)\left(\gamma^{6}+9 \gamma^{4} n^{2}+159 \gamma^{2} n^{4}+367 \gamma^{6}\right)}{\left(n^{2}+\gamma^{2}\right)\left(255 n^{6}+91 \gamma^{2} n^{4}+11 \gamma^{4} n^{2}+\gamma^{6}\right)} & \frac{8 n\left(720 n^{8}+613 n^{6} \gamma^{2}+111 \gamma^{4} n^{4}+3 \gamma^{6} n^{2}+\gamma^{8}\right)}{\gamma\left(n^{2}+\gamma^{2}\right)\left(255 n^{6}+91 \gamma^{2} n^{4}+11 \gamma^{4} n^{2}+\gamma^{6}\right)} \\
\frac{-8 n \gamma^{3}\left(55 n^{4}+20 \gamma^{2} n^{2}+\gamma^{4}\right)}{255 n^{6}+91 \gamma^{2} n^{4}+11 \gamma^{4} n^{2}+\gamma^{6}} & \frac{-4 \gamma^{2}\left(75 n^{6}-7 \gamma^{2} n^{4}-11 \gamma^{4} n^{2}-\gamma^{6}\right)}{255 n^{6}+91 \gamma^{2} n^{4}+11 \gamma^{4} n^{2}+\gamma^{6}}
\end{array}\right] \\
& P_{33}=\left[\begin{array}{cc}
\frac{8\left(45 n^{8}+388 n^{6} \gamma^{2}+138 \gamma^{4} n^{4}+12 \gamma^{6} n^{2}+\gamma^{8}\right)}{\gamma\left(n^{2}+\gamma^{2}\right)\left(255 n^{6}+91 \gamma^{2} n^{4}+11 \gamma^{4} n^{2}+\gamma^{6}\right)} & \frac{48 n^{3}\left(47 n^{4}+10 \gamma^{2} n^{2}-\gamma^{4}\right)}{\gamma^{2}\left(n^{2}+\gamma^{2}\right)\left(255 n^{6}+91 \gamma^{2} n^{4}+11 \gamma^{4} n^{2}+\gamma^{6}\right)} \\
\frac{48 n^{3}\left(47 n^{4}+10 \gamma^{2} n^{2}-\gamma^{4}\right)}{\gamma^{2}\left(n^{2}+\gamma^{2}\right)\left(255 n^{6}+91 \gamma^{2} n^{4}+11 \gamma^{4} n^{2}+\gamma^{6}\right)} & \frac{8\left(405 n^{8}+244 n^{6} \gamma^{2}+66 \gamma^{4} n^{4}+12 \gamma^{6} n^{2}+\gamma^{8}\right)}{\gamma\left(n^{2}+\gamma^{2}\right)\left(255 n^{6}+91 \gamma^{2} n^{4}+11 \gamma^{4} n^{2}+\gamma^{6}\right)}
\end{array}\right] .
\end{aligned}
$$
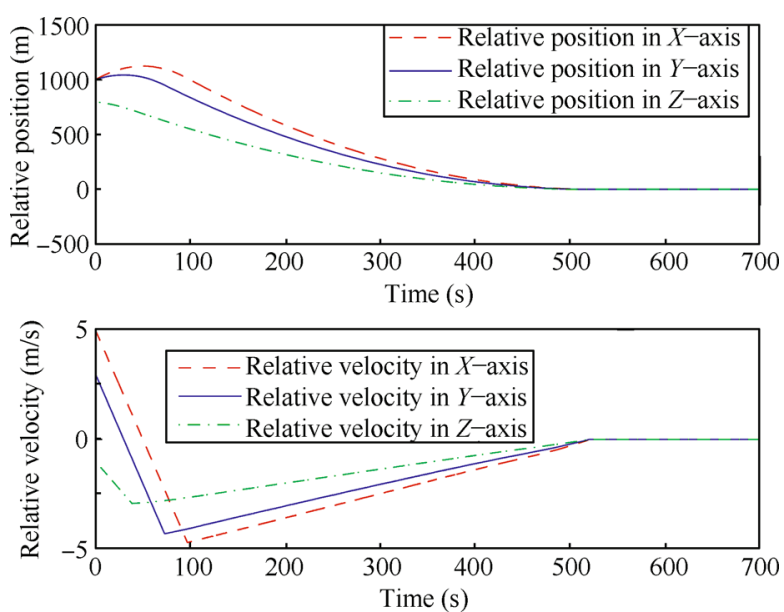

Fig. 2 Relative positions and velocities in $\mathrm{X}, \mathrm{Y}$ and $\mathrm{Z}$ axes
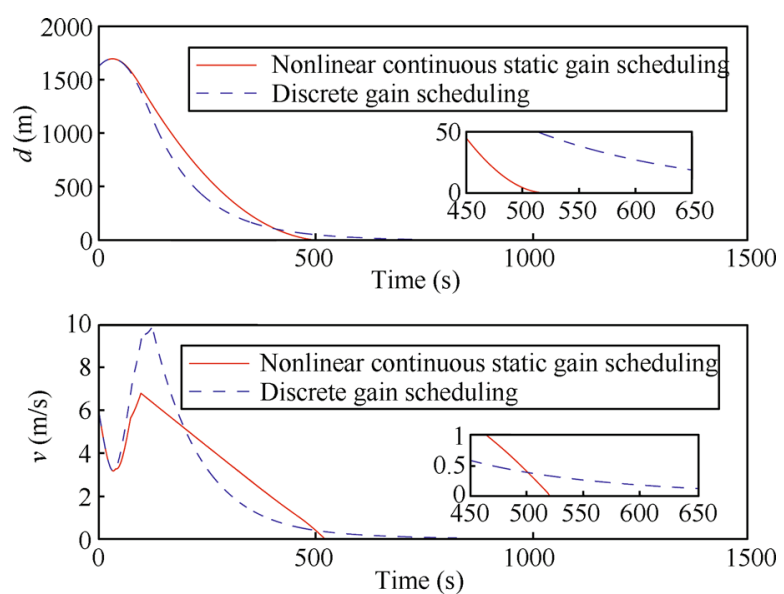

Fig. 3 Relative distances and velocities under the two different control methods, $d=\sqrt{x^{2}+y^{2}+z^{2}}$ and $v=\sqrt{\dot{x}^{2}+\dot{y}^{2}+\dot{z}^{2}}$

As we can see from Figs. 2 and 3, the closed-loop system is asymptotically stable. Actually, the rendezvous mission is accomplished at about $t_{f}=520 \mathrm{~s}$. Fig. 4 gives the curve of control accelerations, which shows that the control input does not exceed the maximal acceleration supplied by the thrusts. Fig. 5 shows the performance of the state trajectories when using controller (11) and also illustrates the ellipsoid boundary $\partial \mathscr{E}$, in which the continuous controller in (11) switches from a nonlinear feedback to a linear one. At $\mathscr{E}$, the closed-loop system behaves as a linear system. According to (12), parameter $\gamma(\boldsymbol{x})$ is changed in $\left(0, \gamma^{*}\right]$ (here $\left.\gamma^{*}=1\right)$, which can be verified by the history of $\gamma(\boldsymbol{x})$ recorded in Fig. 6. We also give the curve of $\eta(\boldsymbol{x})$ in Fig. 6.

We compare the proposed nonlinear continuous static gain scheduling controller with the discrete gain scheduling control established in [28]. Compared with the discrete gain scheduling method, the simulation result in Fig. 3 shows the proposed approach can save about $500 \mathrm{~s}$ for accomplishing the rendezvous mission. The proposed method has a better dynamic performance.

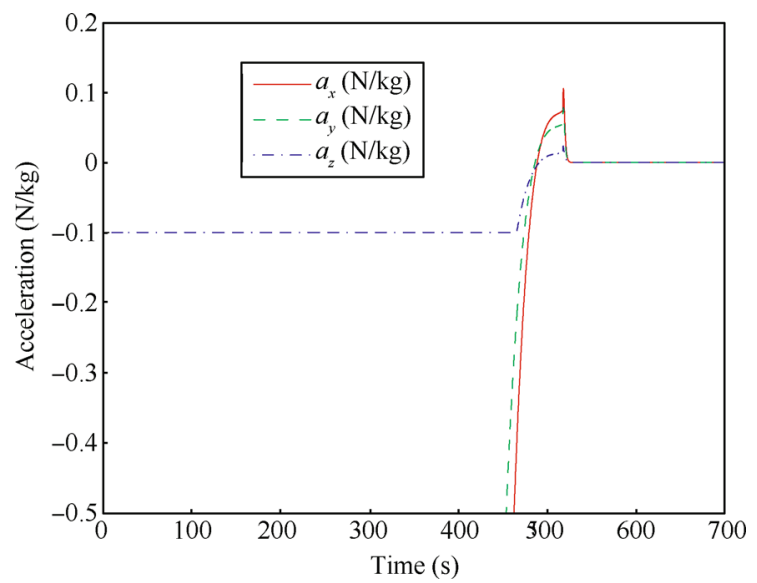

Fig. 4 Control accelerations

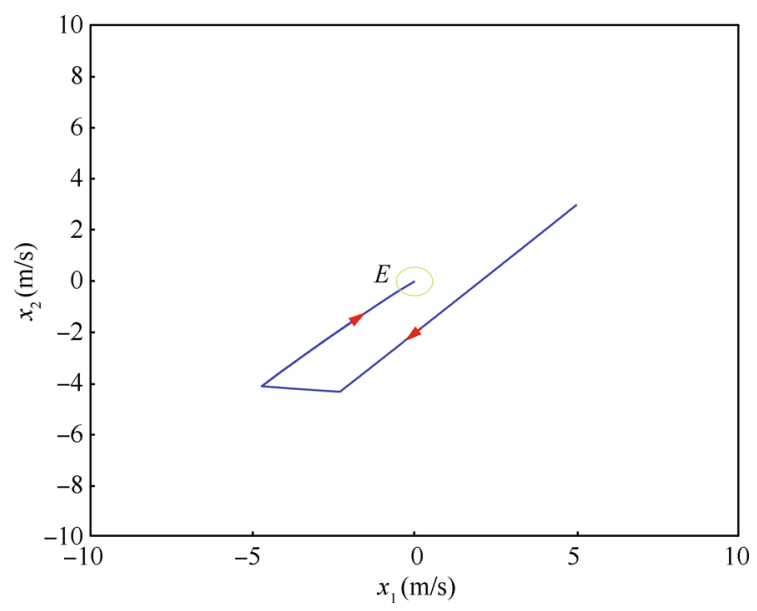

Fig. 5 State trajectory 

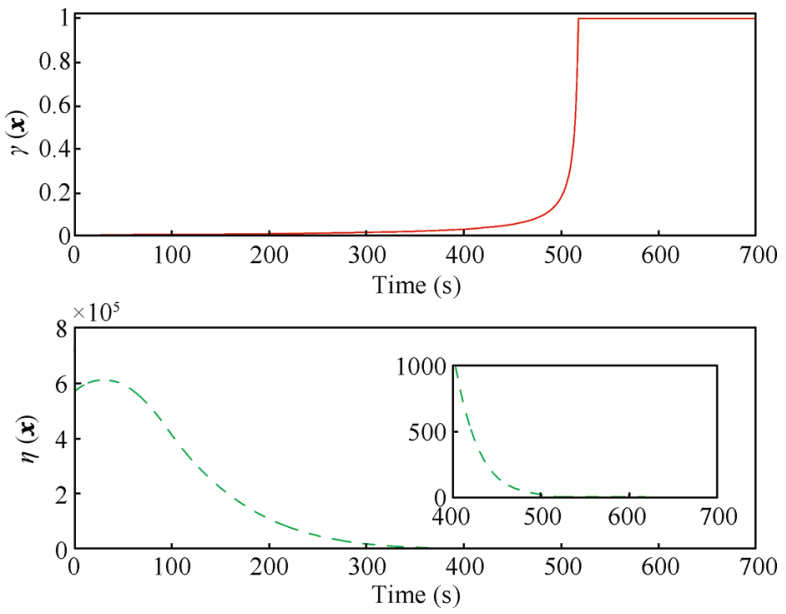

Fig. $6 \quad \gamma(\boldsymbol{x})$ and $\eta(\boldsymbol{x})$

\section{Conclusions}

This paper has proposed a robust gain scheduling approach based on the parametric Lyapunov equation to solve the global stabilization problem of the spacecraft rendezvous system with input saturation and input-additive uncertainties. The designed robust gain scheduling controller is very easy to implement since only a scalar nonlinear equation is required to be solved. Numerical simulations of the nonlinear model of the spacecraft rendezvous operation instead of a linearized one show the proposed robust gain scheduling method finishes the rendezvous mission successfully.

\section{References}

[1] M. E. Polites. Technology of automated rendezvous and capture in space. Journal of Spacecraft and Rockets, vol. 36, no. 2, pp. 280-291, 1999.

[2] W. H. Clohessy, R. S. Wiltshire. Terminal guidance system for satellite rendezvous. Journal of the Aerospace Sciences, vol. 27 , no. 9, pp. 653-658, 1960.

[3] L. S. Breger, J. P. How. Safe trajectories for autonomous rendezvous of spacecraft. Journal of Guidance, Control, and Dynamics, vol. 31, no. 5, pp. 1478-1489, 2008.

[4] A. Richards, J. How. Performance evaluation of rendezvous using model predictive control. In Proceedings of the AIAA Guidance, Navigation, and Control Conference and Exhibit, AIAA, Austin, Texas, USA, pp. 1-9, 2003.

[5] E. Hartley. Model Predictive Control for Spacecraft Rendezvous, Ph. D. dissertation, University of Cambridge, UK, 2010.

[6] L. Singh, S. Bortolami, L. A. Page. Optimal guidance and thruster control in orbital approach and rendezvous for docking using model predictive control. In Proceedings of the AIAA Guidance, Navigation, and Control Conference, AIAA, Toronto, Ontario, Canada, pp. 2010-7754, 2010.

[7] F. Gavilan, R. Vazquez, E. F. Camacho. Chanceconstrained model predictive control for spacecraft rendezvous with disturbance estimation. Control Engineering Practice, vol. 20, no. 2, pp. 111-122, 2012.
[8] P. Singa, K. Subbarao, J. L. Junkins. Adaptive output feedback control for spacecraft rendezvous and docking under measurement uncertainty. Journal of Guidance, Control, and Dynamics, vol. 29, no. 4, pp. 892-902, 2006.

[9] A. Tiwari, J. Fung, J. M. Carson, R. Bhattacharya, R. M. Murray. A framework for Lyapunov certificates for multivehicle rendezvous problems. In Proceedings of 2004 American Control Conference, IEEE, Boston, Massachusetts, pp. 5582-5587, 2004.

[10] E. A. Youmans, F. H. Lutze. Neural network control of space vehicle intercept and rendezvous maneuvers. Journal of Guidance, Control, and Dynamics, vol. 21, no. 1, pp. 116$121,1998$.

[11] F. Zhang, G. R. Duan. Integrated relative position and attitude control of spacecraft in proximity operation missions. International Journal of Automation and Computing, vol. 9, no. 4, pp. 342-351, 2012.

[12] B. Zhou, Z. L. Lin, G. R. Duan. Lyapunov differential equation approach to elliptical orbital rendezvous with constrained controls. Journal of Guidance, Control, and Dynamics, vol. 34, no. 2, pp. 345-358, 2011.

[13] T. E. Carter, J. Brientt. Fuel-optimal rendezvous for linearized equations of motion. Journal of Guidance, Control, and Dynamics, vol. 15, no. 6, pp. 1411-1416, 1992.

[14] T. E. Carter, M. Humi. Fuel-optimal rendezvous near a point in general Keplerian orbit. Journal of Guidance, Control, and Dynamics, vol. 10, no. 6, pp. 567-573, 1987.

[15] Z. L. Lin. Global control of linear systems with saturating actuators. Automatica, vol. 34, no. 7, pp. 897-905, 1998.

[16] A. Saberi, Z. L. Lin, A. R. Teel. Control of linear systems with saturating actuators. IEEE Transactions on $\mathrm{Au}$ tomatic Control, vol. 41, no. 3, pp. 368-378, 1996.

[17] D. J. Leith, W. E. Leithead. Survey of gain-scheduling analysis and design. International Journal of Control, vol. 73, no. 11, pp. 1001-1025, 2000.

[18] W. J. Rugh, J. S. Shamma. Research on gain scheduling. Automatica, vol. 36, pp. 1401-1425, 2000.

[19] Q. Wang, B. Zhou, G. Duan. Global stabilization of spacecraft rendezvous system subject to input saturation via gain scheduling. In Proceedings of the 32nd Chinese Control Conference, Xi'an, China, pp. 1436-1441, 2013.

[20] R. Suárez, J. Álvarez-Ramírez, J. Solís-Daun. Linear systems with bounded inputs: Global stabilization with eigenvalue placement. International Journal of Robust and Nonlinear Control, vol. 7, no. 9, pp. 835-845, 1997.

[21] A. Megretski. Output feedback stabilization with saturated control. In Proceedings of the 13th IFAC World Congress, IFAC, San Francisco, CA, USA, pp. 435-440, 1996.

[22] T. S. Hu, Z. L. Lin. On improving the performance with bounded continuous feedback laws. IEEE Transactions on Automatic Control, vol. 47, no. 9, pp. 1570-1575, 2002.

[23] F. Grognard, R. Sepulchre, G. Bastin. Improving the performance of low-gain designs for bounded control of linear systems. Automatica, vol. 38, no. 10, pp. 1777-1782, 2002.

[24] A. R. Teel. Linear systems with input nonlinearities: Global stabilization by scheduling a family of $H_{\infty}$-type controllers. International Journal of Robust and Nonlinear Control, vol. 5, no. 5, pp. 399-411, 1995.

[25] B. Zhou, Z. L. Lin, G. R. Duan. Robust global stabilization of linear systems with input saturation via gain scheduling. International Journal of Robust and Nonlinear Control, vol. 20 , no. 4 , pp. 424-447, 2010. 
[26] J. A. De Dona, S. O. Reza Moheimani, G. C. Goodwin, A. Feuer. Robust hybrid control incorporating over-saturation. Systems \& Control Letters, vol. 38, no. 3, pp. 179-185, 1999.

[27] T. S. Hu, Z. L. Lin, Y. Shamash. Semi-global stabilization with guaranteed regional performance of linear systems subject to actuator saturation. Systems \& Control Letters, vol. 43, no. 3, pp. 203-210, 2001.

[28] G. F. Wredenhagen, P. R. Bélanger. Piecewise-linear LQ control for systems with input constraints. Automatica, vol. 30, no. 3, pp. 403-416, 1994.

[29] T. E. Carter. State transition matrices for terminal rendezvous studies: Brief survey and new example. Journal of Guidance, Control, and Dynamics, vol.21, no. 1, pp.148$155,1998$.

[30] Z. L. Lin, A. Saberi. A semi-global low-and-high gain design technique for linear systems with input saturationstabilization and disturbance rejection. International Journal of Robust and Nonlinear Control, vol. 5, no. 5, pp. 381398, 1995.

[31] Z. L. Lin, A. Saberi. Semi-global exponential stabilization of linear systems subject to input saturation via linear feedbacks. Systems \& Control Letters, vol. 21, no. 3, pp. 225239, 1993.

[32] Z. L. Lin, A. A. Stoorvoge, A. Saberi. Output regulation for linear systems subject to input saturation. Automatica, vol. 32, no. 1, pp. 29-47, 1996.

[33] A. R. Teel. Semi-global stabilizability of linear null controllable systems with input nonlinearities. IEEE Transactions on Automatic Control, vol. 40, no. 1, pp.96-100, 1995.
[34] B. Zhou, G. R. Duan, Z. L. Lin. A parametric Lyapunov equation approach to the design of low gain feedback. IEEE Transactions on Automatic Control, vol. 53, no. 6, pp. 15481554, 2008.

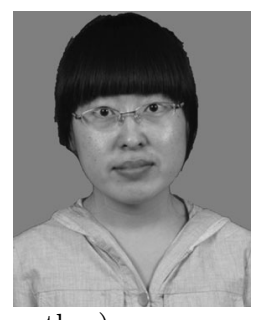

author)

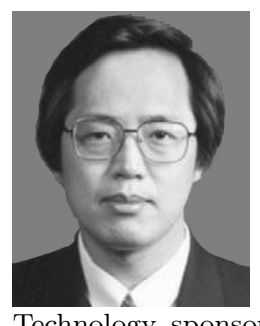

Qian Wang is currently a Ph.D. candidate of Center for Control Theory and Guidance Technology at Harbin Institute of Technology.

Her research interests include gain scheduling control, systems with saturated nonlinearity, and control of spacecraft rendezvous system.

E-mail: wq@hit.edu.cn (Corresponding

Guang-Ren Duan received his B.Sc. degree in applied mathematics, and both his M. Sc. and Ph. D. degrees in control systems theory. From 1989 to 1991, he was a post-doctoral researcher at Harbin Institute of Technology, where he became a professor of control systems theory in 1991. Since August 2000, he has been elected specially employed professor at Harbin Institute of Technology sponsored by the Cheung Kong Scholars Program of the Chinese government. He is currently the director of the Center for Control Theory and Guidance Technology at Harbin Institute of Technology. He is a Chartered Engineer in the UK, a senior member of IEEE and a fellow of IEE.

His research interests include robust control, eigenstructure assignment, descriptor systems, missile autopilot control and magnetic bearing control.

E-mail: g.r.duan@hit.edu.cn 